\title{
MetaGenyo: a web tool for meta-analysis of genetic association studies
}

\author{
Jordi Martorell-Marugan ${ }^{1}$, Daniel Toro-Dominguez ${ }^{1,2}$, Marta E. Alarcon-Riquelme ${ }^{2,3}$ and Pedro Carmona-Saez ${ }^{1 *}$ (D)
}

\begin{abstract}
Background: Genetic association studies (GAS) aims to evaluate the association between genetic variants and phenotypes. In the last few years, the number of this type of study has increased exponentially, but the results are not always reproducible due to experimental designs, low sample sizes and other methodological errors. In this field, meta-analysis techniques are becoming very popular tools to combine results across studies to increase statistical power and to resolve discrepancies in genetic association studies. A meta-analysis summarizes research findings, increases statistical power and enables the identification of genuine associations between genotypes and phenotypes. Meta-analysis techniques are increasingly used in GAS, but it is also increasing the amount of published meta-analysis containing different errors. Although there are several software packages that implement meta-analysis, none of them are specifically designed for genetic association studies and in most cases their use requires advanced programming or scripting expertise.

Results: We have developed MetaGenyo, a web tool for meta-analysis in GAS. MetaGenyo implements a complete and comprehensive workflow that can be executed in an easy-to-use environment without programming knowledge. MetaGenyo has been developed to guide users through the main steps of a GAS meta-analysis, covering Hardy-Weinberg test, statistical association for different genetic models, analysis of heterogeneity, testing for publication bias, subgroup analysis and robustness testing of the results.
\end{abstract}

Conclusions: MetaGenyo is a useful tool to conduct comprehensive genetic association meta-analysis. The application is freely available at http://bioinfo.genyo.es/metagenyo/.

Keywords: Genetic association study, Meta-analysis, Web tool, Shiny

\section{Background}

Genetic association studies (GAS) estimate the statistical association between genetic variants and a given phenotype, usually complex diseases [1]. In the last few years, the number of genetic association studies has increased exponentially, but the results are not consistently reproducible. This lack of reproducibility may be influenced by several factors, including the analysis of non-heritable phenotype, inappropriate quality control, wrong statistical analysis, low sample size, population stratification, incorrect multiple-testing correction or technical biases [2].

Meta-analysis is a statistical technique for combining results across studies and it is becoming very popular as a method for resolving discrepancies in GAS. It summarizes

\footnotetext{
* Correspondence: pedro.carmona@genyo.es

'Bioinformatics Unit, Centre for Genomics and Oncological Research

(GENYO), Granada, Spain

Full list of author information is available at the end of the article
}

research findings, increases statistical power and enables the identification of genuine associations [3]. In this context, in 2011 there was a 64-fold increase in geneticsrelated meta-analysis compared to 1995 [4].

Despite the increasing number of publications in this field there is a lack of dedicated software tools to perform a complete GAS meta-analysis in a friendly environment. In this context, most published works in the field have used commercial software suites such as STATA [5] or SPSS [6]. These are statistical software packages that include general functions for meta-analysis in their configuration. In addition, freely available $\mathrm{R}$ packages such as meta [7] or metafor [8] are also widely used but all these solutions share common limitations: do not provide all required steps for a GAS meta-analysis (e.g. evaluating Hardy Weinberg equilibrium (HWE) or genetic models) and require advanced statistical or bioinformatics knowledge to be properly used. 
In this context, Park et al. have reported several analytical errors in published GAS meta-analysis [9], many of them could be avoided using a dedicated software for GAS meta-analysis with predefined functions and automatic computations of the required statistics.

Here we present MetaGenyo, an easy-to-use web application which implements a complete meta-analysis workflow for GAS. Once the data has been loaded, it provides a guided and complete workflow that comprises the main steps in GAS meta-analysis, including HWE test, checking heterogeneity, publication bias indicators, statistical association testing for different genetics models, subgroup analysis and robustness testing. The use of MetaGenyo does not require advanced statistical or bioinformatics knowledge and we hope it will be a useful application for researchers working in the field of genetic association studies.

\section{Implementation}

MetaGenyo has been implemented as a web tool using shiny [10], a web application framework for RStudio [11]. Backend computations are carried out in R using available packages and custom scripts. MetaGenyo provides the following functionalities:

\section{Testing HWE}

Departures from HWE can occur due to genotyping errors, selection bias and stratification [12]. Therefore, goodness-of-fit of HWE should be checked in each study before pooling data. HardyWeinberg package [13, 14] is used to compute a $P$-value for each study in the control population in order to identify low-quality studies. As we test for HWE in several studies, the obtained $P$ values are corrected by Benjamini and Hochberg false discovery rate (FDR) [15].

\section{Genetic models}

Given two alleles (A, a) the three possible genotypes (AA, Aa, aa) can be dichotomized in different ways yielding different genetic models. GAS can be carried out assuming a specific genetic model based on biological criteria but in most of the cases different models are simultaneously evaluated. MetaGenyo performs meta-analysis in several ways [16], including allele contrast (A vs. a), recessive (AA vs. Aa + aa), dominant (AA + Aa vs. aa) and overdominant (Aa vs. AA + aa) genetic models as well as pairwise comparisons (AA vs. aa, AA vs. Aa and Aa vs. aa). All $P$-values are adjusted for multiple testing with the Bonferroni method [17].

\section{Statistical analysis and heterogeneity}

To perform meta-analysis, MetaGenyo combines the effect sizes of the included studies by weighting the data according to the amount of information in each study.
Association values are calculated based on two different statistic models: Fixed Effects Model (FEM) and Random Effects Model (REM). The choosing between both models depends on the amount of heterogeneity in the data, which is also evaluated with heterogeneity indicators such as $\mathrm{I}^{2}$ and Cochran's Q test (see on-line help of the program). Meta package (7) is used to get such heterogeneity indicators and association results. Finally, this same package is used to generate forest plots to summarize information for effect size and the corresponding 95\% confidence interval (CI) of each study and the pooled effect. Forest plots can be generated for FEM, REM or both, and can be downloaded with very high resolution.

\section{Publication bias}

Publication bias occurs because of meta-analysis are performed using published studies, which usually report only significant associations, while studies showing no significant results tend to remain unpublished. This may therefore give a falsely skewed positive result. To test for publication bias, MetaGenyo provides funnel plots and Egger's test [16] for each genetic model. Funnel plots are generated with meta package [7] and Egger's test is performed using the metafor package [8].

\section{Subgroup analysis}

MetaGenyo provides a subgroup analysis in order to evaluate associations in a subset of studies based on the user defined criteria (e.g. studies from the same country). Many genetic associations are population-specific and can be undiscovered in a general meta-analysis, but discovered when studies are split. For each group, a meta-analysis is performed with FEM or REM, depending on the heterogeneity test: If heterogeneity $P$-value $<0.1, R E M$ will be used. Otherwise, FEM will be used instead. These results are downloadable in Excel and text formats.

\section{Sensitivity analysis}

In order to test the robustness of the meta-analysis performed, MetaGenyo performs a leave-one-out influence analysis using meta package [7]. Briefly, the meta-analysis is repeated several times, each time excluding one of the studies, in order to determine how each individual study affects the overall statistics [18]. A forest plot with these results is generated for the selected genetic model.

\section{Software usage}

An overview of MetaGenyo is provided in the on-line help of the application and Fig. 1. First, the user loads the collected data from individual studies as a text or Excel file with some specifications on the file format. Once the data has been loaded, a complete analysis is performed providing results and visualizations in different 




tabs: (1) The data tab, where the user can check if the data has been correctly submitted. (2) Hardy-Weinberg tab, where a HWE $P$-value column is added to the data. (3) Association values tab. This contains different association values and heterogeneity indicators for each genetic model. (4) Forest plot tab contains forest plot visualizations in high-quality image format for each genetic model. (5) Publication bias tab, where the user can see the funnel plot and Egger's test results. (6) Subgroup analysis tab to obtain a summary of the analysis or to evaluate the association and heterogeneity results taking into account stratification based on user-defined variables and, finally, (7) Sensitivity tab to perform a robustness analysis.

\section{Results and discussion}

Despite there are many programs designed to perform genome-wide association studies (GWAS) meta-analysis (reviewed in [19]), there is a lack of tools specially designed to perform GAS meta-analysis, so researchers use general statistical or meta-analysis software, adapting it to the particular purposes in such type of metaanalysis. This lack of dedicated software increases the required resources to perform a GAS meta-analysis, facilitates the inclusion of methodological errors and requires advanced bioinformatics expertise.

Among the most widely used software solutions in this field are STATA [5], SPSS [6] and SAS [20]. These are popular software suites that provide a set of statistical functions that can be used in a broad range of applications and data analysis problems, but they are proprietary software and are not specialized in GAS metaanalysis. These limitations are partially overcome by $\mathrm{R}$ packages such as meta [7], rmeta [21] and metafor [8]. These are freely-available software libraries to perform a complete meta-analysis in a flexible way. However, their use requires $\mathrm{R}$ programming skills, they do not provide a guided workflow and they are not specifically designed to perform GAS meta-analysis. In addition, there are some Excel extensions such as MIX [22] and MetaEasy [23]. These extensions are easy to use, but they require the usage of the proprietary software Microsoft Excel.

In this context, MetaGenyo is a user-friendly web application that implements a complete meta-analysis following a guided workflow, which does not require programming knowledge. Table 1 contains a summary of the main advantages and disadvantages of some reviewed GAS meta-analysis software.

To demonstrate the functionality of MetaGenyo we have used data from a published GAS meta-analysis [24]. In this study, the authors performed a meta-analysis to study the association between the A23G SNP of XPA gene (rs1800975) and digestive cancers. They collected genotype information from 18 case-control studies including 4170 patients and 6929 controls in total. In this polymorphism, the $\mathrm{G}$ allele was considered the reference, so the A allele was the risk allele (this parameter must be specified in MetaGenyo). Results from the complete analysis and a comparison with results reported in the original article can be found in Additional file 1.

Briefly, both sets of results are highly concordant, but in the original publication the authors did not correct the $P$ values for multiple testing or evaluated different genetic models as provided by MetaGenyo. We found some discrepancies between both sets of results due to use of inappropriate statistical tests or labeling mistakes, especially at the subgroup analysis step (see Additional file 1). Because MetaGenyo automatically performs all metaanalysis steps in a guided analysis we reduced these potential sources of errors. All these similarities and differences are detailed in Additional file 1.

The application generated results for all possible genetic models and allowed us to easily evaluate results for 
Table 1 Characteristics of available meta-analysis software

\begin{tabular}{|c|c|c|c|c|c|c|c|c|}
\hline & STATA & SPSS & MIX & MetaEasy & meta & rmeta & metafor & MetaGenyo \\
\hline \multicolumn{9}{|l|}{ USABILITY } \\
\hline Availability & Commercial & Commercial & Commercial $^{a}$ & Free $^{b}$ & Free & Free & Free & Free \\
\hline Web-based & No & No & No & No & No & No & No & Yes \\
\hline Operating system & $\begin{array}{l}\text { Windows, Mac } \\
\text { OS, Linux }\end{array}$ & $\begin{array}{l}\text { Windows, Mac } \\
\text { OS, Linux }\end{array}$ & Windows & Windows & $\begin{array}{l}\text { Windows, Mac } \\
\text { OS, Linux }\end{array}$ & $\begin{array}{l}\text { Windows, Mac } \\
\text { OS, Linux }\end{array}$ & $\begin{array}{l}\text { Windows, Mac } \\
\text { OS, Linux }\end{array}$ & Any $^{c}$ \\
\hline Guided workflow & No & No & No & No & No & No & No & Yes \\
\hline $\begin{array}{l}\text { Programming } \\
\text { knowledge }\end{array}$ & Yes $^{d}$ & Yes $^{d}$ & No & No & $\mathrm{R}$ language & $\mathrm{R}$ language & $\mathrm{R}$ language & No \\
\hline \multicolumn{9}{|l|}{ FUNCTIONALITIES } \\
\hline $\begin{array}{l}\text { Specific for GAS } \\
\text { meta-analysis }\end{array}$ & No & No & No & No & No & No & No & Yes \\
\hline HWE testing & Yes & No & No & No & No & No & No & Yes \\
\hline $\begin{array}{l}\text { Heterogeneity } \\
\text { assessment }\end{array}$ & Yes & Yes & Yes & Yes & Yes & Yes & Yes & Yes \\
\hline $\begin{array}{l}\text { Random/Fixed effect } \\
\text { models }\end{array}$ & Yes & Yes & Yes & Yes & Yes & Yes & Yes & Yes \\
\hline Forest plot & Yes & Yes & Yes & Yes & Yes & Yes & Yes & Yes \\
\hline $\begin{array}{l}\text { Automatic testing } \\
\text { of genetic models }\end{array}$ & No & No & No & No & No & No & No & Yes \\
\hline Publication bias & Yes & Yes & Yes & No & Yes & Yes & Yes & Yes \\
\hline Subgroup analysis & Yes & No & Yes & No & Yes & No & Yes & Yes \\
\hline Robustness analysis & Yes & No & Yes & No & Yes & No & Yes & Yes \\
\hline $\begin{array}{l}\text { P-value correction } \\
\text { for multiple testing }\end{array}$ & Yes & Yes & No & No & No & No & No & Yes \\
\hline
\end{tabular}

${ }^{a}$ There is a MIX free version with reduced capabilities. ${ }^{b}$ MetaEasy is free, but it depends on the proprietary software Microsoft Excel. ${ }^{c}$ MetaGenyo is accessed through an internet browser, so there are no limitations regarding the operating system used to access it. ${ }^{d}$ Although STATA and SPSS are command-based software, there are graphical user interfaces (GUIs) available which permits replacing scripting by user-friendly interactive commands

different subgroups in a unified framework. In this context, using the tumor type feature to stratify the data revealed a significant association for the overdominant model in esophageal cancer studies not previously reported $(\mathrm{OR}=0.83,95 \% \mathrm{CI}=0.74-0.93, P$-value $=0.0016$, Bonferroni-adjusted P-value $=0.0448$ ) [Fig. 2]. Although the original work reported no significant association between this polymorphism and the risk of any type of digestive cancer for the studied models, there may be a protective effect of AG genotype against the risk of esophageal tumors overlooked at the original article because the authors did not test this genetic model. Indeed, a similar association has been found in another GAS meta-analysis with lung cancer samples [25].

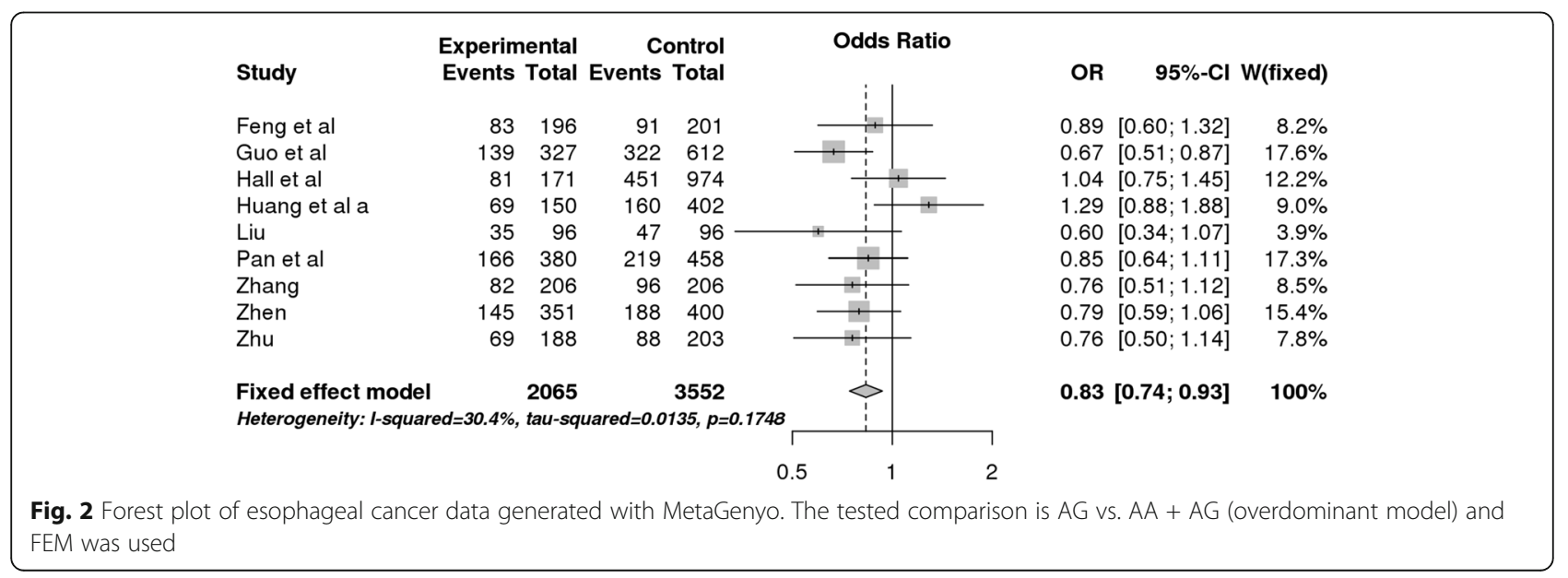




\section{Conclusions}

In this work, we present MetaGenyo, a free easy-to-use web tool to perform GAS meta-analysis. It provides a guided workflow through the most important steps of a meta-analysis.

We demonstrated MetaGenyo's functionality replicating a previously published meta-analysis [24]. In addition, thanks to the automatic testing of several genetic models and subgroup analysis we found a significant association between rs1800975 SNP in XPA gene and esophageal cancer under the overdominant genetic model that may be interesting enough for further testing.

Surprisingly, there is a large heterogeneity in statistical methods, lack of quality control steps or misleading reporting and interpretation of results in many published meta-analysis [9]. Therefore, an application such as MetaGenyo will be a very useful tool for the research community providing a guided and solid workflow.

\section{Availability}

Project name: MetaGenyo.

Availability: MetaGenyo web tool, example datasets and help are accessible at http://bioinfo.genyo.es/metagenyo/.

Any restrictions on use by academics: none.

\section{Additional file}

Additional file 1: MetaGenyo's use case. Document showing the results of analyzing the data provided by [24] using MetaGenyo and comparison with the original results. (PDF $253 \mathrm{~kb}$ )

\section{Abbreviations}

Cl: Confidence intervals; FDR: False discovery rate; FEM: Fixed effect model; GAS: Genetic association study; GUI: Graphical user interface; GWAS: Genome-wide association study; HWE: Hardy-Weinberg equilibrium; OR: Odds-ratio; REM: Random effect model; $x^{2}$ : Goodness-of-fit chi-square

\section{Acknowledgements}

We thank Alberto Ramirez and Manuel Martinez for helpful technical assistance.

\section{Funding}

JMM is partially supported by Ministerio de Economía y Competitividad [grant number PEJ 2014-A-95230].

\section{Availability of data and materials}

All data generated or analyzed during this study are included in this published article and its supplementary information files.

\section{Author's contributions}

PCS conceived the project and directed the software development. JMM designed the software and performed the analysis. DTD and MAR tested the software, provided improvements and test cases. PCS and JMM wrote the manuscripts. All authors read and approved the final manuscript.

\section{Ethics approval and consent to participate} Not applicable.
Consent for publication

Not applicable.

\section{Competing interests}

The authors declare that they have no competing interests.

\section{Publisher's Note}

Springer Nature remains neutral with regard to jurisdictional claims in published maps and institutional affiliations.

\section{Author details}

${ }^{1}$ Bioinformatics Unit, Centre for Genomics and Oncological Research (GENYO), Granada, Spain. ${ }^{2}$ Medical Genomics, Centre for Genomics and Oncological Research (GENYO), Granada, Spain. ${ }^{3}$ Institute for Environmental Medicine, Karolinska Institutet, Stockholm, Sweden.

Received: 9 August 2017 Accepted: 6 December 2017

Published online: 16 December 2017

References

1. Cardon LR, Bell Jl. Association study designs for complex diseases. Nat Rev Genet. 2001 Feb;2(2):91-9.

2. Li A, Meyre D. Challenges in reproducibility of genetic association studies: lessons learned from the obesity field. Int J Obes. 2005. 2013 Apr;37(4):55967.

3. Trikalinos TA, Salanti G, Zintzaras E, loannidis JPA. Meta-analysis methods. Adv Genet. 2008;60:311-34.

4. Ioannidis JPA, Chang CQ, Lam TK, Schully SD, Khoury MJ. The geometric increase in meta-analyses from China in the genomic era. PLoS One. 2013; 8(6):e65602.

5. StataCorp. Stata Statistical Software. College Station, TX: StataCorp LP; 2015.

6. IBM Corp. SPSS statistics for windows. IBM Corp: Armonk, NY; 2016.

7. Schwarzer G. Meta: an R package for meta-analysis. R News. 2007;7(3):40-6.

8. Viechtbauer W. Conducting meta-analyses in R with the metafor package. J Stat Softw. 2010;36(3):1-48

9. Park JH, Eisenhut M, van der Vliet HJ, Shin JI. Statistical controversies in clinical research: overlap and errors in the meta-analyses of microRNA genetic association studies in cancers. Ann Oncol Off J Eur Soc Med Oncol. 2017 Jun 1;28(6):1169-82

10. Chang W, Cheng J, Allaire JJ, Xie Y, McPherson J. shiny: Web Application Framework for R [Internet]. 2017. Available from: https://CRAN.R-project.org/ package=shiny

11. RStudio Team. RStudio: integrated development for R [internet]. RStudio Inc Boston, MA; 2015. Available from: http://www.rstudio.com/

12. Salanti G, Amountza G, Ntzani EE, loannidis JPA. Hardy-Weinberg equilibrium in genetic association studies: an empirical evaluation of reporting, deviations, and power. Eur J Hum Genet EJHG. 2005 Jul;13(7): 840-8.

13. Graffelman J. Exploring Diallelic genetic markers: the HardyWeinberg package. J Stat Softw. 2015:64:1-22.

14. Graffelman J, Camarena JM. Graphical tests for hardy-Weinberg equilibrium based on the ternary plot. Hum Hered. 2008;65(2):77-84.

15. Benjamini Y, Hochberg Y. Controlling the false discovery rate: a practical and powerful approach to multiple testing. J R Stat Soc. 1995;57(1):289-300.

16. Attia J, Thakkinstian A, D'Este C. Meta-analyses of molecular association studies: methodologic lessons for genetic epidemiology. J Clin Epidemiol. 2003 Apr:56(4):297-303.

17. Bonferronin CE. Teoria statistica delle classi e calcolo delle probabilità. Pubblicazioni del R Istituto Superiore di Scienze Economiche e Commerciali di Firenze. 1936;

18. Viechtbauer W, MW-L C. Outlier and influence diagnostics for meta-analysis. Res Synth Methods. 2010 Apr;1(2):112-25.

19. Begum F, Ghosh D, Tseng GC, Feingold E. Comprehensive literature review and statistical considerations for GWAS meta-analysis. Nucleic Acids Res. 2012 May;40(9):3777-84

20. SAS Institute Inc. SAS. Cary, NC: SAS Institute Inc; 2011.

21. Lumley T. rmeta: Meta-analysis [Internet]. 2012. Available from: https://CRAN R-project.org/package=rmeta

22. Bax L, L-M Y, Ikeda N, Tsuruta H, Moons KG. Development and validation of MIX: comprehensive free software for meta-analysis of causal research data. BMC Med Res Methodol. 2006 Oct 13;6:50. 
23. Kontopantelis E, Reeves D. MetaEasy: a meta-analysis add-in for Microsoft excel. J Stat Softw. 2009;30(7)

24. He L, Deng T, Luo H. XPA A23G polymorphism and risk of digestive system cancers: a meta-analysis. OncoTargets Ther. 2015;8:385-94.

25. Liu X, Lin Q, Fu C, Liu C, Zhu F, Liu Z, et al. Association between XPA gene rs 1800975 polymorphism and susceptibility to lung cancer: a meta-analysis. Clin Respir J. 2016 Jul;27

Submit your next manuscript to BioMed Central and we will help you at every step:

- We accept pre-submission inquiries

- Our selector tool helps you to find the most relevant journal

- We provide round the clock customer support

- Convenient online submission

- Thorough peer review

- Inclusion in PubMed and all major indexing services

- Maximum visibility for your research

Submit your manuscript at www.biomedcentral.com/submit 